Bond University

Research Repository

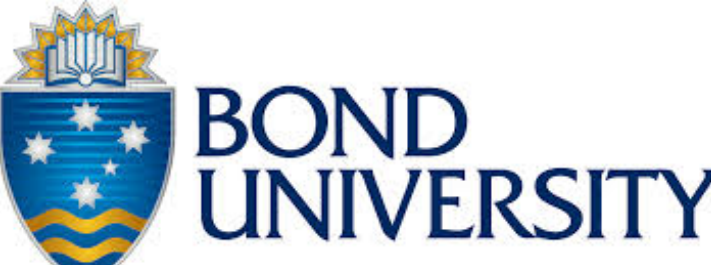

\section{Framework to Enhance Teaching and Learning in System Analysis and Unified Modelling Language}

Munoz, Juan Carlos; Cowling, Michael A.; Birt, James R.

Published in:

Proceedings of 2018 IEEE International Conference on Teaching, Assessment, and Learning for Engineering, TALE 2018

DOI:

10.1109/TALE.2018.8615284

10.1109/TALE.2018.8615284

Licence:

Free to read

Link to output in Bond University research repository.

Recommended citation $(A P A)$ :

Munoz, J. C., Cowling, M. A., \& Birt, J. R. (2018). Framework to Enhance Teaching and Learning in System Analysis and Unified Modelling Language. In M. J. W. Lee, S. Nikolic, G. K. W. Wong, J. Shen, M. Ros, L. C. U. Lei, \& N. Venkatarayalu (Eds.), Proceedings of 2018 IEEE International Conference on Teaching, Assessment, and Learning for Engineering, TALE 2018 (pp. 91-98). [8615284] (Proceedings of IEEE International Conference on Teaching Assessment and Learning for Engineering). IEEE Computer Society.

https://doi.org/10.1109/TALE.2018.8615284, https://doi.org/10.1109/TALE.2018.8615284

\section{General rights}

Copyright and moral rights for the publications made accessible in the public portal are retained by the authors and/or other copyright owners and it is a condition of accessing publications that users recognise and abide by the legal requirements associated with these rights.

For more information, or if you believe that this document breaches copyright, please contact the Bond University research repository coordinator. 


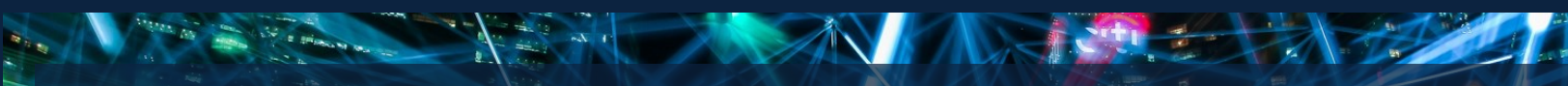 \\ IEEE TALE 2018}

Engineering Next-Generation Learning

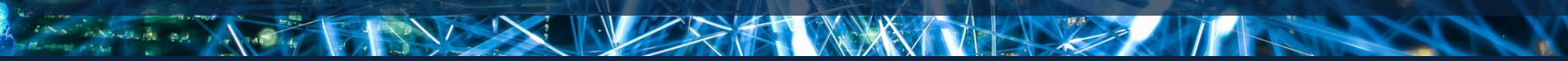

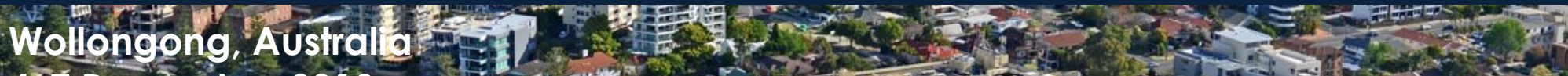

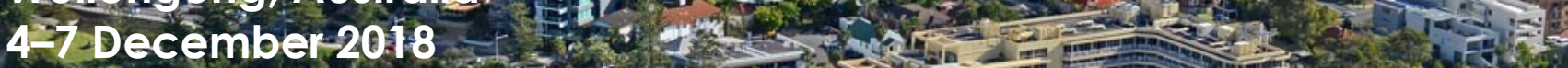
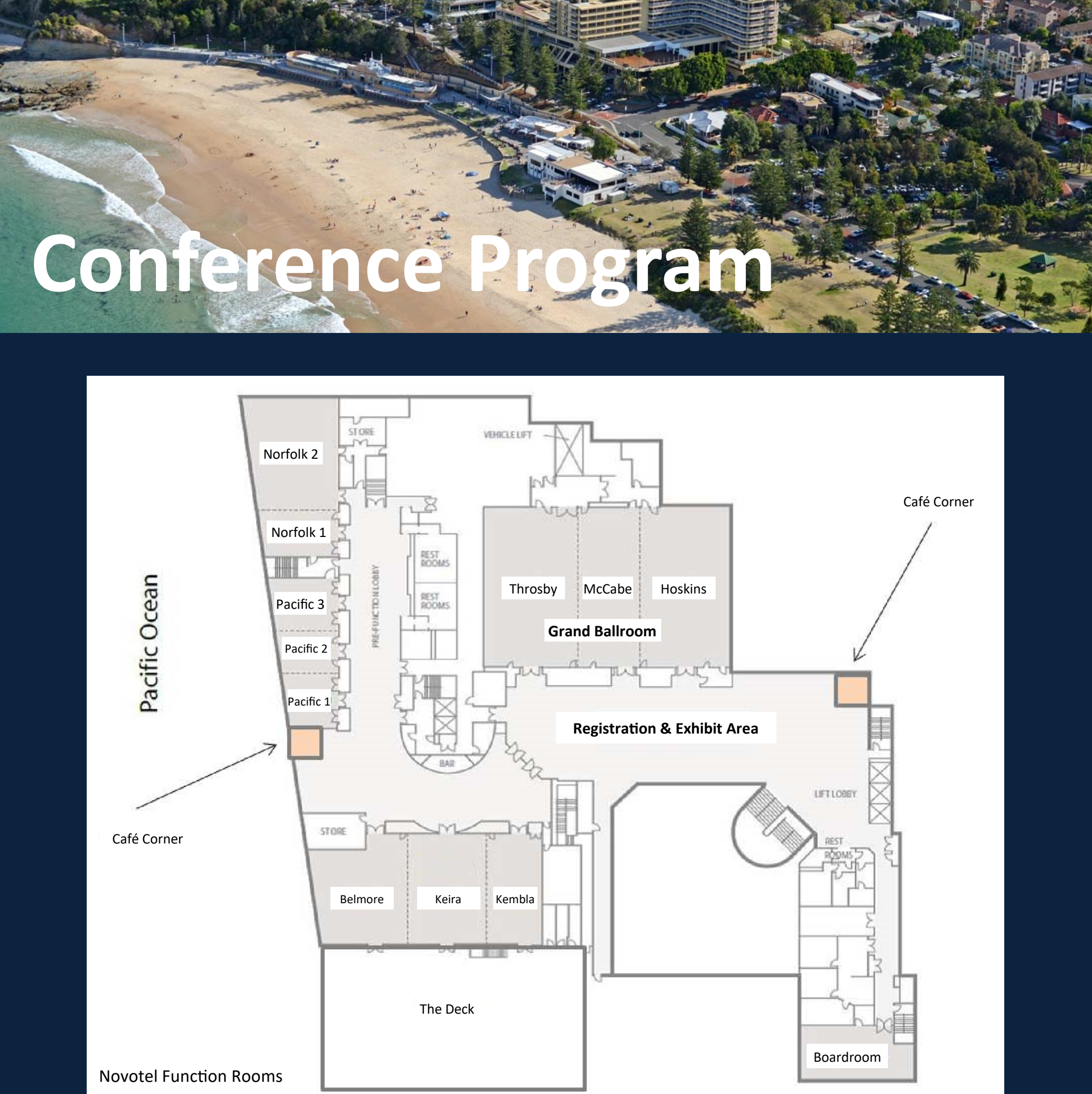


\section{Tuesday, 4 Dec 2018}

\section{Day \#1: University of Wollongong Campus}

$11: 30-19: 30$
$12: 00-15: 00$
$12: 00-15: 00$
$12: 00-15: 00$
$12: 00-15: 00$
$12: 00-15: 00$
$16: 00-16: 45$
$17: 00-19: 10$
$19: 10-21: 30$

Registration

Pre-Conference Workshop 1: Patents \& IP Law

Pre-Conference Workshop 2: Analytics \& Student Support

Pre-Conference Workshop 3: Remote Labs

Pre-Conference Workshop 4 - Chatbot Tutors

Pre-Conference Workshop 5: XR \& Immersive Learning

UOW Campus Tour

Conference Opening with Keynotes by Susan Zhang \& Dr Bror Saxberg

Welcome Reception
UOW Campus - Hope Theatre

UOW Campus - Building 17, Room 106

UOW Campus - Building 3, Room 122

UOW Campus - Building 4, Room 122

UOW Campus - Building 4, Room 123

UOW Campus - Building 6, Room 210

Meet at UOW Campus - Building 4 Atrium

UOW Campus - Hope Theatre

UOW Campus - UniBar

\section{Wednesday, 5 Dec 2018}

\section{Day \#2: Novotel Wollongong Northbeach}

$\begin{array}{lll}\text { 08:30-17:00 } & \text { Registration } & \text { Registration \& Exhibit Area } \\ \text { 09:00-10:10 } & \text { Opening Session with Keynote by Prof Gregor Kennedy } & \text { Grand Ballroom } \\ \text { 10:10-16:30 } & \text { VR/AR Demo Showcase } & \text { Norfolk } 1 \text { \& } 2 \\ \text { 10:10-10:40 } & \text { Poster Session } & \text { Registration \& Exhibit Area } \\ \mathbf{1 0 : 3 0 - 1 0 : 5 5} & \text { Morning Tea } & \text { Registration \& Exhibit Area }\end{array}$

Throsby (Ballroom 1) : Engineering Education - Full Papers (10min presentations + 3min questions)

\begin{tabular}{|c|c|c|c|c|}
\hline Time & Sub. ID & Contribution Tit & Family Name / First Name / Presenter Affiliation & Country \\
\hline \multirow[t]{2}{*}{ 11:00-11:13 } & 244 & \multicolumn{3}{|c|}{ Enhancing Teaching Methods on Embedded Systems with Project-Based Learning } \\
\hline & & Sanfilippo & Norwegian University of Science and Technology & Norway \\
\hline \multirow[t]{2}{*}{$11: 15-11: 28$} & 306 & \multicolumn{3}{|c|}{ Contribution of Learning, Teaching and Assessment Activities to the Development of 21st Century STEM Competencies } \\
\hline & & Hettling & Brandenburg University of Technology Cottbus-Senftenberg & Germany \\
\hline \multirow[t]{2}{*}{$11: 30-11: 43$} & 284 & \multicolumn{3}{|c|}{ The Link between Spatial Skills and Engineering Problem-Solving } \\
\hline & & Sheryl & University of Cincinnati & USA \\
\hline \multirow[t]{2}{*}{$11: 45-11: 58$} & 227 & \multicolumn{3}{|c|}{ Evaluating Teaching Effectiveness Using Quantitative Student Feedback } \\
\hline & & Srinivasan & National University of Singapore & Singapore \\
\hline \multirow[t]{2}{*}{ 12:00-12:13 } & 395 & \multicolumn{3}{|c|}{ Using Problem-Based Learning to Enable Application of Foundation Engineering Knowledge in a ReatWorld Problem } \\
\hline & & Larson & Arizona State University & USA \\
\hline
\end{tabular}

\section{McCabe (Ballroom 2) : Computing \& IT Education - Full Papers (10min presentations + 3min questions)}

\begin{tabular}{|c|c|c|}
\hline Time & Sub. ID & Contribution Title | Presenter Family Name / First Name / Presenter Affiliation \\
\hline \multirow[t]{2}{*}{$11: 00-11: 13$} & 134 & A Quantitative Approach to Design Special Purpose Systems to Measure Hacking Skills \\
\hline & & Deakin University \\
\hline \multirow[t]{2}{*}{$11: 15-11: 28$} & 138 & Educating and Raising Awareness on Cyber Security Social Engineering: A Literature Review \\
\hline & & University of Newcastle \\
\hline \multirow[t]{2}{*}{$11: 30-11: 43$} & 359 & Approach for Evaluating IT Employees' Programming Ability Using the Programed Visual Contents Comparison Method \\
\hline & & Kobe University \\
\hline \multirow[t]{2}{*}{$11: 45-11: 58$} & 366 & Teaching Hardware Reverse Engineering: Educational Guidelines and Practical Insights \\
\hline & & Ruhr-Universität Bochum \\
\hline \multirow[t]{2}{*}{$12: 00-12: 13$} & 467 & An Essential Applied Statistical Analysis Course using RStudio with Project-Based Learning for Data Science \\
\hline & & Singapore Management University \\
\hline
\end{tabular}

\section{Country}

Australia

Australia

Japan

Germany

Singapore

Hoskins (Ballroom 3) : Technology Enhanced Learning - Full Papers (10min presentations + 3min questions)

\section{Time \\ $11: 00-11: 13$ \\ Sub. ID \\ 125}

$11: 15-11: 28$

184

11:30-11:43 275

$11: 45-11: 58 \quad 371$

$12: 00-12: 13$

\section{Contribution Title / Presenter Family Name / First Name / Presenter Affiliation}

Country Application and Evaluation of a Grouped Flipped Classroom Method

Umezawa Katsuyuki Shonan Institute of Technology

Japan

Peer Assisted Study Sessions (PASS) Online: Investigating the impact of an online format across different first year university subjects

Woolrych University of Wollongong

Australia

Students Discussing Ideas in Online Spaces: Research-Infused Recommendations for Making Computer-Mediated Discussions Productive for Learning

Zengilowski Allison Nicole University of Texas at Austin

USA

Evaluation of Developing Educational Chatbots Based on The Seven Principles for Good Teaching

Luo Jing The University of Hong Kong

Hong Kong

Transferring Human Tutor's Style to Pedagogical Agent: A Possible Way by Leveraging Variety of Artificial Intelligence

Achievements

Feng

Xiang

East China Normal University

China 
Kembla : STEM Education (K-12) Full Papers - Full Papers (10min presentations + 3min questions)

\section{Time}

11:00-11:13

Sub. ID

270

$11: 15-11: 28 \quad 105$

$11: 30-11: 43 \quad 157$

$11: 45-11: 58$

318

$12: 00-12: 13 \quad 337$

Contribution Title | Presenter Family Name / First Name / Presenter Affiliation

Country

Using Maths Model Method in Solving Pre Algebraic Problems among Year Five Students

Binti Ismail Zaleha Universiti Teknologi Malaysia

Malaysia

Arduino as an Educational Tool to Introduce Robotics
Castro
Manuel
UNED

Spain

Computational Thinking and Coding Subject in Primary Schools: Methodological Approach Based on Alternative Cooperative and Individual Learning Cycles

Vlahu-Gjorgievska Elena University of Wollongong

Australia

Computational Thinking Education for Children: Algorithmic Thinking and Debugging

Wong Gary K. W. The University of Hong Kong

Hong Kong

Framework of Integrating Algebraic Thinking in Problem-Based Learning via Online Environment for School Students Binti Ismai

Zaleha

Universiti Teknologi Malaysia

Malaysia

\section{Pacific 182: XR \& Immersive Learning Environments - Full Papers (10min presentations + 3min questions)}

Time

11:45-11:58 391

$12: 00-12: 13 \quad 444$

Contribution Title | Presenter Family Name / First Name / Presenter Affiliation

Country

Design and Implementation of an Augmented Reality Application with an English Learning Lesson

Liu Enrui Beijing Normal University

China

Investigating Using Behaviors of E-dictionary with Multiple Design: A Perspective from the Integration of Eye-Tracking Technique and Stimulated Recall

Zhai Xuesong $\{$ Michael\} Beijing Normal University

China

\section{Pacific 3 : Engineering Education - Full Papers (10min presentations + $3 \mathrm{~min}$ questions)}

\section{Time}

\section{Sub. ID}

Contribution Title | Presente

Country

Are We Fit to Graduate Creative Professionals?

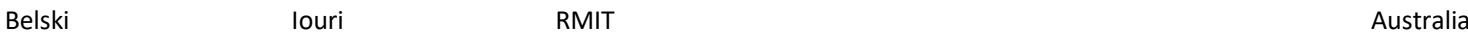

11:15-11:28 $474 \quad$ Teaching Reform of Engineering Graphics Education in Terms of Engineering Education Professional Certification in Guangzhou, China
Liu
Zhen
South China University of Technology
China

$11: 30-11: 43 \quad 486$

Learning Strategies to Optimize the Assimilation of ITC2 Competencies for Business Engineering Programs
Luna
Ana
Universidad del Pacífico
Peru

A Java Program for Automatic Team Allocation in Project-Based Coursework
Vial
Peter James
University of Wollongong

Australia

Higher Education Practices Accounting for Gender Differences in Technological Development as Part of SDGs

Achievement

Hirata Sadayo Shibaura Institute of Technology

Japan

\section{Keira : Industry Sessions}

\begin{tabular}{|c|c|c|c|c|}
\hline Time & Sub. ID & Contribution Titl & Family Name / First Name / Presenter Affiliation & Country \\
\hline \multirow[t]{2}{*}{$11: 00-11: 40$} & 500 & Microsoft HoloLens and Al & & \\
\hline & & Townes & Microsoft & Australia \\
\hline \multirow[t]{2}{*}{$11: 40-12: 00$} & 505 & Enriching Education: Free Classro & Education Resources Increase STEM Diversity & \\
\hline & & Damian & Oracle Academy & Australia \\
\hline
\end{tabular}

Belmore: Panel Session

Time Sub. ID

Contribution Title | Presenter Family Name / First Name / Presenter Affiliation

Country

11:00-12:20 $500 \quad$ Panel Session - Providing Automated and Individually Tailored Assessment Feedback

$\begin{array}{llll}\text { Cunningham-Nelson } & \text { Samuel } & \text { Queensland University of Technology } & \text { Australia } \\ \text { Mohammadi-Aragh } & \text { Jean } & \text { Mohammadi-Aragh } & \text { USA } \\ \text { Goncher } & \text { Andrea } & \text { Charles Sturt University } & \text { Australia } \\ \text { Boles } & \text { Wageeh } & \text { Queensland University of Technology } & \text { Australia }\end{array}$

Registration and Exhibit Area and Norfolk Rooms \& Windjammers Restaurant 
Time

Sub. ID

$14: 00-14: 13$

Contribution Title | Presenter Family Name / First Name / Presenter Affiliation

Country

Mind the Gap: Insights into Student Perceptions During Peer Assessment of Writing

Tan Benjamin The University of Auckland

New Zealand

Dawood Rahmad Universitas Syiah Kuala Indonesia

14:30-14:43 $461 \quad$ Objective Assessment of Students' Generic Skills

Yajima Kuniaki National Institute of Technology, Sendai College Japan

$14: 45-14: 58 \quad 306$

An Inter-Disciplinary and University PBL Curriculum Using Robot Challenge

Hisazumi Kenji Kyushu University Japan

15:00-15:13 $322 \quad$ Exploring the Impact of In-Class Writing Exercises in an Engineering Course

Nadeem Muhammad The University of Auckland New Zealand

15:15-15:28 $227 \quad$ Creative Thinking of Engineering Undergraduates through Brainstorming during Mathematical Problem Solving

Mohammad Yusof Yudariah Universiti Teknologi Malaysia Malaysia

McCabe (Ballroom 2) : Computing \& IT Education - Full Papers (10min presentations + 3min questions)

\begin{tabular}{|c|c|c|c|}
\hline Time & Sub. ID & Contribution Title I Presenter Family Name / First Name / Presenter Affiliation & Coun \\
\hline \multirow[t]{2}{*}{$14: 00-14: 13$} & 467 & A Comparison Analysis between Achievement and Requirements for Computing Education & \\
\hline & & Saga University & Japan \\
\hline \multirow[t]{3}{*}{$14: 15-14: 28$} & 347 & Skill-Based Group Allocation of Students for Project-Based Learning Courses Using Genetic Algorithm: Weighted & \\
\hline & & Penalty Model & \\
\hline & & The University of the South Pacific & Fiji \\
\hline \multirow[t]{3}{*}{$14: 30-14: 43$} & 365 & Skill-Based Group Allocation of Students for Project-Based Learning Courses Using Genetic Algorithm: Weightless & \\
\hline & & Penalty Model & \\
\hline & & The University of the South Pacific & Fiji \\
\hline \multirow[t]{2}{*}{$14: 45-14: 58$} & 384 & Effectiveness of Physical Robot Versus Robot Simulator in Teaching Introductory Programming & \\
\hline & & Singapore University of Technology and Design & Singapore \\
\hline \multirow[t]{2}{*}{$15: 00-15: 13$} & 130 & SplashKit: A Development Framework for Motivating and Engaging Students in Introductory Programming & \\
\hline & & Deakin University & Australia \\
\hline \multirow[t]{2}{*}{$15: 15-15: 28$} & 294 & Improving outcomes for a Masters Capstone IT Project & \\
\hline & & The University of Adelaide & Australia \\
\hline
\end{tabular}

Hoskins (Ballroom 3) : Technology-Enhanced Learning - Full Papers (10min presentations + 3min questions)

Time Sub. ID

14:00-14:13 272

$14: 15-14: 28 \quad 446$

$14: 30-14: 43 \quad 194$

$14: 45-14: 58 \quad 271$

$15: 00-15: 13 \quad 288$

$15: 15-15: 28 \quad 320$
Contribution Title | Presenter Family Name / First Name / Presenter Affiliation

Country

Technology-Supported Single Training for One-on-One in Basketball Matches

Kohda Naoya Tokushima University

Japan

A Gamification Approach For Serious Games

Carron Thibault LIP6, UPMC

France

An Empirical Study on the Impact of Pre-recorded Lectures on Students' Performance in Integral Calculus

Fernando Alexa Ray Ronsairo National University

Philippines

Enhanced Student Learning in Proteomics - An Interactive Tool Support for Teaching Workflows

Wickramarachchi Anuradha Australian National University

Australia

Making the Most of Repetitive Mistakes: An Investigation into Heuristics for Selecting and Applying Feedback to

Programming Coursework

Howell Roger Aston University

UK

The Effectiveness of Integrating Geometer's Sketchpad Software in Phase-Based Geometric Learning

Mohd Zaid Norasykin Universiti Teknologi Malaysia

Kembla : STEM Education (K-12) - Full Papers (10min presentations + 3min questions)

\section{Time Sub. ID \\ Contribution Title | Presenter Family Name / First Name / Presenter Affiliation}

$14 \cdot 00-14: 13 \quad 374$

Country

Relationship between Teachers' Self-Efficacy and Instructional Strategies Applied among Secondary School Teachers in Implementing STEM Education

Binti Ismail Zaleha Universiti Teknologi Malaysia

Malaysia

14:15-14:28 $114 \quad$ Relationships between Collaborative Problem Solving, Learning Performance and Learning Behavior in Science Education

Chen Li Kyushu University

14:30-14:43 $212 \quad$ Cognitive Load Optimisation - A New, Practical, Easy-to-Use Teaching Method for Enhancing STEM Educational Outcomes Based on the Science of Learning

Maj Stanislaw Paul

SPM Consulting

$14: 45-14: 58 \quad 285$

$15: 00-15: 13 \quad 439$

$15: 15-15: 28 \quad 277$

Pandian Shunmugham R. Indian Institute of Information Technology, Design and Manufacturing India ICT Competency, Network Interaction, Internet Self-efficacy, and Mathematical Achievement: Direct and Mediating Effects
Lu
Chun
Central China Normal University

China

Awakening the Interest of High School Pupils in Science, Technology, Engineering and Mathematics Studies and Careers through Scientific Projects 


\begin{tabular}{|c|c|c|}
\hline Time & Sub. ID & Contribution Title | Presenter Family Name / First Name / Presenter Affiliation \\
\hline \multirow[t]{2}{*}{ 14:00-14:13 } & 258 & Evaluation of a Virtual Reality Nasal Cavity Education Tool \\
\hline & & Auckland University of Technology \\
\hline \multirow[t]{3}{*}{ 14:15-14:28 } & 482 & Immersive Learning Explored: Subjective and Objective Factors Influencing Learning Outcomes in Immersive \\
\hline & & Educational Virtual Environments \\
\hline & & University of Passau \\
\hline \multirow[t]{2}{*}{ 14:30-14:43 } & 313 & Integration of Virtual Reality and Augmented Reality: Are They Worth the Effort in Education? \\
\hline & & Kent Institute \\
\hline \multirow[t]{2}{*}{ 14:45-14:58 } & 353 & Collaborative Virtual Reality Simulation for Multi-Professional Training in Emergency Management Communications \\
\hline & & Norwegian University of Science and Technology \\
\hline \multirow[t]{2}{*}{ 15:00-15:13 } & 355 & $\begin{array}{l}\text { Developing Virtual Reality Open Educational Resources in a Sino-Foreign Higher Education Institution: Challenges and } \\
\text { Strategies }\end{array}$ \\
\hline & & University of Nottingham Ningbo China \\
\hline \multirow[t]{2}{*}{ 15:15-15:28 } & 380 & $\begin{array}{l}\text { A Study on Female Students' Attitude Towards the Use of Augmented Reality to Learn Atoms and Molecules Reactions } \\
\text { in a Palestinian School }\end{array}$ \\
\hline & & Arab American University \\
\hline
\end{tabular}

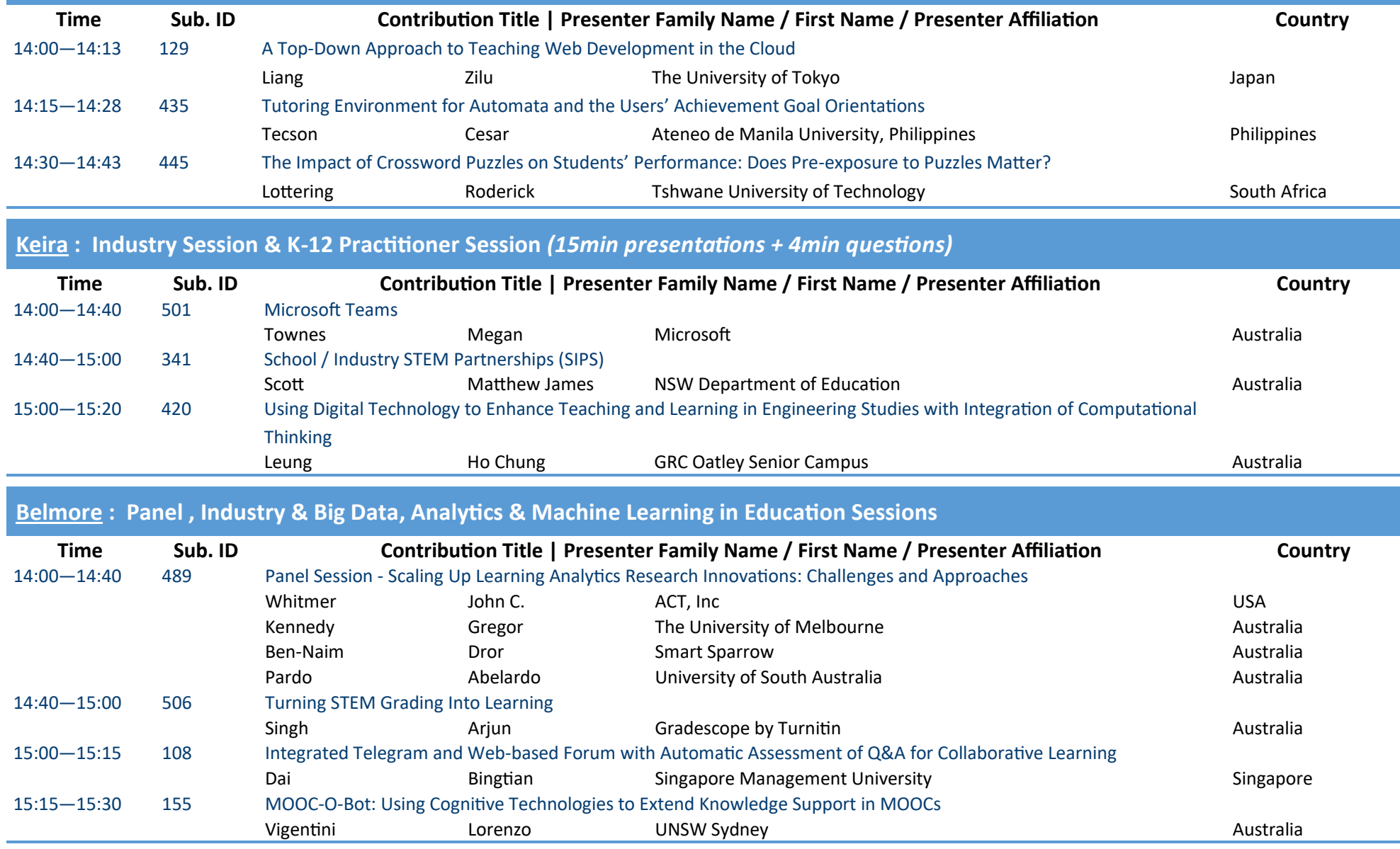

Registration \& Exhibit Area

15:55-15:55

Throsby (Ballroom 1) : Engineering Education - Short Papers ( 6 min presentations + 2 min questions)

$\begin{array}{cc}\text { Time } & \text { Sub. ID } \\ \text { 16:00-16:08 } & 214 \\ & \\ 16: 10-16: 18 & 316\end{array}$

\section{Contribution Title | Presenter Family Name / First Name / Presenter Affiliation}

Country

A Holistic View on Engineering Education: How to Educate Control Engineers

Samuelsen Dag A. H University of Southeastern Norway

Norway

Smart Grid Education: A Review of Global Course Offerings and the UNSW Sydney Approach

Konstantinou Georgios University of New South Wales

Australia

From an International Classroom to a Distributed Work Environment: Student Perspectives on Global Software Engineering

Vasilache Simona University of Tsukuba

Japan

Unplugged Game Play Motivates the Study of Engineering Ethics

Assessment Map for Multidisciplinary Abilities for loT System Development Education [abased on a Robot Contest

Watanabe Harumi Tokai University


McCabe (Ballroom 2) : Big Data, Analytics \& Machine Learning in Education and Industry Sessions

Time

Sub. ID

16:00-16:08 245

$16: 10-16: 18 \quad 263$

$16: 20-16: 28 \quad 415$

$16: 30-16: 38 \quad 416$

$16: 40-17: 00 \quad 506$

\section{Contribution Title | Presenter Family Name / First Name / Presenter Affiliation}

Country

Artificial Intelligence to Enhance Learning Design in UOW Online, a Unified Approach to Fully Online Learning

Cao Trinh University of Wollongong

Australia

Using Data Mining and Machine Learning Approaches to Observe Technology-Enhanced Learning

Howard Sarah Katherine University of Wollongong

Australia

Inferring the Climate in Classrooms from Audio and Video Recordings: A Machine Learning Approach

Kashyap Mohan Nanyang Technological University

Singapore

Hybrid Fuzzy-Statistical System for Learning Analytics

Sohail Shaleeza King's Own Institute Australia

Turning STEM Grading Into Learning (Repeat)

Singh Arjun Gradescope by Turnitin

Australia

Hoskins (Ballroom 3) : Technology-Enhanced Learning - Short Papers (6min presentations + 2min questions)

Time Sub. ID

16:00-16:08 436

$16: 10-16: 18-150$

$16: 20-16: 28 \quad 225$

$16: 30-16: 38 \quad 273$

$16: 40-16: 53 \quad 329$

\section{Contribution Title | Presenter Family Name / First Name / Presenter Affiliation}

Developing Higher Order Thinking Skill with the 120-Minute Instructional Station Rotation (MRSP120) Approach: Students' Perceptions

Mohd Zaid Norasykin Universiti Teknologi Malaysia Malaysia

Usability Testing of VLASTA: A Vocabulary Learning and Strategy Teaching App
Mirzaei
Siamak
Flinders Univesity
Australia

An Innovative Hybrid Model for Developing Cross Domain ICT Talent in Digital Economy

Wu Hsin-jung Institute for Information Industry

Taiwan

Improving Student's Engagement through the Use of Learning Modules, Instantaneous Feedback and Automated Marking

Rattadilok Prapa University of Nottingham Ningbo China

China

Improving Feedback and Discussion in MOOC Peer Assessment using Introduced Peers - Full Paper (10min pres $+3 \mathrm{~min}$ ) Gamage

Dilrukshi

University of Moratuwa

Sri Lanka

\section{Kembla : STEM Education (K-12) - Short Papers ( $6 \mathrm{~min}$ presentations + $2 \mathrm{~min}$ questions)}

\begin{tabular}{|c|c|c|c|}
\hline Time & Sub. ID & Contribution Title / Presenter Family Name / First Name / Presenter Affiliation & Cou \\
\hline \multirow[t]{2}{*}{$16: 00-16: 08$} & 268 & Supporting Physics Teachers to Deliver the New High School Certificate Syllabus: What are the Priorities? & \\
\hline & & University of Wollongong & Australia \\
\hline \multirow[t]{2}{*}{$16: 10-16: 18$} & 390 & Teaching Mathematics with Music: A Pilot Study & \\
\hline & & Macquarie University & Australia \\
\hline \multirow[t]{2}{*}{$16: 20-16: 28$} & 424 & Kids Making Al : Integrating Machine Learning, Gamification, and Social Context in STEM Education & \\
\hline & & King Mongkut's University of Technology Thonburi & Thailand \\
\hline \multirow[t]{2}{*}{$16: 30-16: 38$} & 428 & Hack Biodesign: An Integrative STEAM Education in Biology, Engineering and Design & \\
\hline & & Naresuan University & Thailand \\
\hline \multirow[t]{2}{*}{$16: 40-16: 48$} & 477 & Preparing Australian High School Learners with 21st Century Skills & \\
\hline & & Central Queensland University & Australia \\
\hline \multirow[t]{2}{*}{$16: 50-16: 58$} & 319 & The Effectiveness of a Mentor-Mentee Program on Malaysian School Students' Interest in STEM & \\
\hline & & Universiti Teknologi Malaysia & Malaysia \\
\hline
\end{tabular}

Pacific 1 \& 2 : XR \& Immersive Learning Environments - Short Papers (6min presentations + 2min questions)

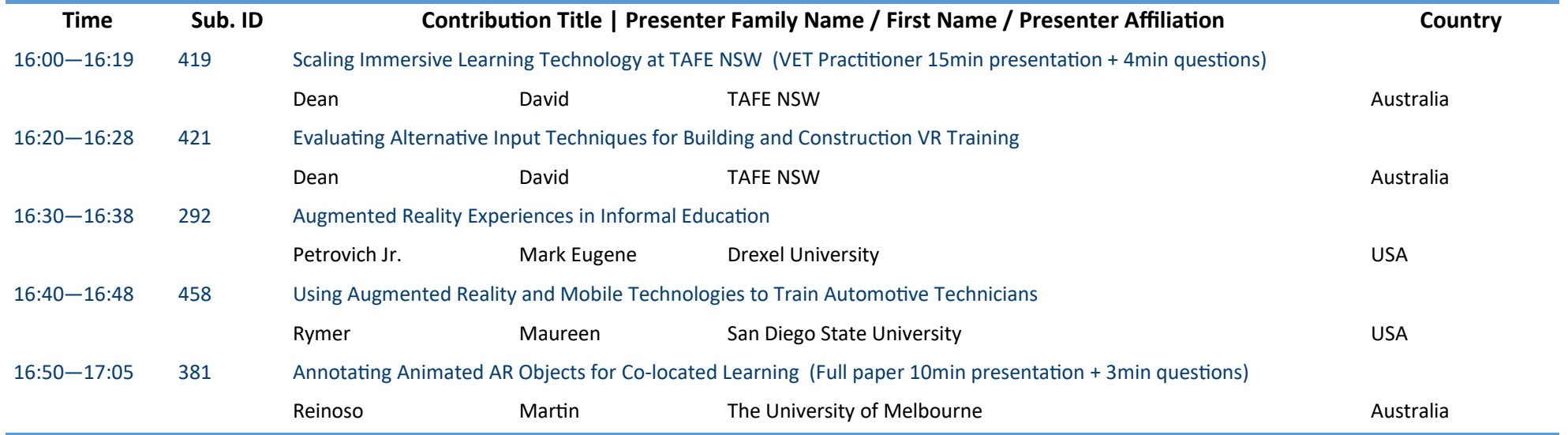

Pacific 3: Industry Session

\begin{tabular}{|c|c|c|c|}
\hline Time & Sub. ID & Contribution T & r Family Name / First Name / Presenter Affiliation \\
\hline \multirow[t]{2}{*}{$16: 00-16: 40$} & 512 & Seamless, Intelligent Mechatro & latform \\
\hline & & Kuttner & 3DS \\
\hline \multirow[t]{2}{*}{$16: 40-17: 20$} & 475 & Scaling Automated Scoring: Ad & I and Conceptual Challenges \\
\hline & & Lazendic & Australian Curriculum, Assessment and Reporting Autho \\
\hline
\end{tabular}

Country

Country

tralia

tralia

iland

iland

tralia 
Panel Session - Learning Technology Standards

$\begin{array}{llll}\text { Wait } & \text { Lisa } & \text { eWorks } & \text { Australia } \\ \text { Chadwick } & \text { Ben } & \text { Education Services Australia } & \text { Australia } \\ \text { Saliah-Hassane } & \text { Hamadou } & \text { TÉLUQ University } & \text { Canada } \\ \text { Jon } & \text { Mason } & \text { Charles Darwin University } & \text { Australia }\end{array}$

\section{Belmore: Panel Session}

Time Sub. ID

Women in Engineering Networking Panel

$\begin{array}{llll}\text { Chakraborty } & \text { Sudipta } & \text { Macquarie University } & \text { Australia } \\ \text { Ros } & \text { Montserrat } & \text { University of Wollongong } & \text { Australia } \\ \text { Cheng } & \text { Eva } & \text { University of Technology, Sydney } & \text { Australia } \\ \text { Goncher } & \text { Andrea } & \text { Charles Sturt University } & \text { Australia } \\ \text { Vial } & \text { Alanna } & \text { University of Wollongong } & \text { Australia }\end{array}$

\section{Thursday, 6 Dec 2018}

\section{Day \#3: Novotel Wollongong Northbeach}

$08: 30-16: 30$

Registration

Registration \& Exhibit Area

Throsby (Ballroom 1) : Engineering Education - Full Papers (10min presentations +3 min questions)

\begin{tabular}{|c|c|c|c|c|}
\hline Time & Sub. ID & Contribution Title & r Family Name / First Name / Presenter Affiliation & Country \\
\hline \multirow[t]{2}{*}{$9: 30-9: 43$} & 251 & Theory And Practice In A Systems In & oject Life Cycle & \\
\hline & & Wolff & University of Stellenbosch & South Africa \\
\hline \multirow[t]{2}{*}{$9: 45-9: 58$} & 470 & Exploring Approaches for Teaching & Engineering Asset Management Courses & \\
\hline & & El-Akruti & University of Wollongong in Dubai & UAE \\
\hline \multirow[t]{2}{*}{ 10:00-10:13 } & 183 & Complete Flipping of a Large Advan & al Course: Engaging Students as Partners & \\
\hline & & Swapneel & UNSW Sydney & Sydney \\
\hline \multirow[t]{2}{*}{$10: 15-10: 28$} & 161 & A Study on The Process and Effect o & erative Learning Approach into Electronics Lecture & \\
\hline & & Pei-Hua & Kun Shan University & Taiwan \\
\hline \multirow[t]{2}{*}{ 10:30-10:43 } & 269 & Copyright Awareness and Video Ass & Case Study on Thai First-Year Engineering Students & \\
\hline & & Maneeratana & Chulalongkorn University & Thailand \\
\hline
\end{tabular}

McCabe (Ballroom 2) : Computing \& IT Education - Full Papers (10min presentations + 3min questions)

Time Sub. ID

$9: 30-9: 43$

$9: 45-9: 58$

172

10:00-10:13 185

$10: 15-10: 28 \quad 213$

$10: 30-10: 43$
Contribution Title | Presenter Family Name / First Name / Presenter Affiliation

Country

Verifying User Concentration Based on Brainwave Control Applied to Different Game Training Methods

Liu Chui Yuan Institute for Information Industry

Taiwan

Framework to Enhance Teaching and Learning in System Analysis and Unified Modelling Language

Muñoz Carpio Juan Carlos Central Queensland University

Australia

International Comparison of College-Level Computing Education

Kakeshita Tetsuro Saga University

Japan

Redesigning a Compulsory Computing Internship Course for Massification

Chng Sue Inn Sunway University

Malaysia

Developing a Language Learning Application OER

Towey

Dave

University of Nottingham Ningbo China

China 
Kembla : Laboratory Learning and Technology-Enhanced Learning - Full Papers (10min presentations $+3 \mathrm{~min}$ questions)

Time

Sub. ID

Contribution Title | Presenter Family Name / First Name / Presenter Affiliation

Country

$9: 30-9: 43$

Applied Learning Through Industry Lab In A Course On RF Engineering \& EMC

Venkatarayalu Neelakantam Singapore Institute of Technology

Singapore

$9: 45-9: 58$

Pedagogy for the Practising Engineer: A Closed Loop Feedback Control Analogy to Teaching and Learning

McLauchlan Craig University of Wollongong

Australia

10:00-10:13

Understanding Common Student Mistakes in the Remote Laboratory NetLab

Considine Hugh University of South Australia

Australia

$10: 15-10: 28$

Smart Learning in the Pacific: Design of New Pedagogical Tools

Nand Ravneil The University of the South Pacific

$10: 30-10: 43$

Evaluation of Automatic Collaborative Learning Process Coding Using Deep Learning Methods

Shibata Chihiro Tokyo University of Technology

Japan

Keira : Open, Flexible \& Workplace Learning - Full Papers (10min presentations + 3min questions)

Time Sub. ID

Contribution Title | Presenter Family Name / First Name / Presenter Affiliation

Country

9:30-9:43 $345 \quad$ Learning Management Systems in the Workplace: A Literature Review

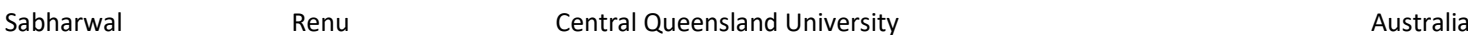

9:45-9:58 $293 \quad$ Student Collegiality in Interdisciplinary Global Contexts

Mitric Damir La Trobe University Australia

10:00-10:13 $405 \quad$ An Intervention Study on the Professional Adaptability of Students in Navigation Course of Educational Technology

Wu Yong he China China Normal University

10:15-10:28 $348 \quad$ Transnational Interactive Blended Learning: A Learning Community of Practice

Wang Minjuan San Diego State University

USA

Belmore : Special \& Industry Sessions

Time Sub. ID

Contribution Title | Presenter Family Name / First Name / Presenter Affiliation

Country

9:30-10:10 $478 \quad$ Designing Virtual Learning Experiences: Iterating Immersive Environments
Dongas
Robert
The University of Sydney
Australia

10:10-10:30 $506 \quad$ Festo/LabVolt Electromechanical Systems - A Practical Demonstration

Lewis Martin Duff and Macintosh-Festo LabVolt

Australia

Boardroom : By Invitation Only

Time Sub. ID

Contribution Title | Presenter Family Name / First Name / Presenter Affiliation

Country

9:30-10:45 N/A TALE Steering Committee Meeting

R10

\section{Registration \& Exhibit Area}
10:45-11:15
Morning Tea
Registration \& Exhibit Area

Throsby (Ballroom 1) : Engineering Education - Short Papers (6min presentations +2 min questions) \& Industry Session

Time

Sub. ID

Contribution Title | Presenter Family Name / First Name / Presenter Affiliation

Country

Hämäläinen Raimo Pertti Aalto University

Finland

11:30-11:38 $\quad 182$

$11: 40-11: 48 \quad 370$

$11: 50-11: 58 \quad 168$

$12: 00-12: 08 \quad 398$

$12: 10-12: 30 \quad 504$
Academic Expectations for Engineering Freshmen: Gender Differences

Vasconcelos Rosa M. University of Minho

Portugal

Active Interdisciplinary Learning in a Design Thinking Course: Going to Class for a Reason

Gillet Denis cole Polytechnique Fédérale de Lausanne

Switzerland

Facilitating the Development of Humor in Electrical Engineering Students

O'Shea Peter The University of Queensland

Australia

Context and Input Evaluations of Physical Fitness Training Programs in Maritime Schools

Bueta $\quad$ Rosalin Duller Maritime Academy of Asia and the Pacific
The Hands-on Engineer: Helping Students Master the Spectrum Analyzer

Tan

Tristen

Rohde \& Schwarz

Australia 
11:20-11:28 $127 \quad$ How We Face Globalization of Chinese Education

Zhao Liang Shenyang Aerospace University China

11:30-11:38 $309 \quad$ Active Learning to Develop Key Research Skills in Master's Level Computer Science Coursework

Carlson Trevor E. National University of Singapore Singapore

11:40-11:48 $401 \quad$ Teaching Key Machine Learning Principles Using Anti-Learning Datasets

Roadknight Chris University of Nottingham Ningbo China China

11:50-11:58 $\quad 430 \quad$ A Proposal for Integrating Gamification into Task-Oriented Portfolio Assessment

Renzella Jake Deakin University Australia

Hoskins (Ballroom 3) : Technology-Enhanced Learning - Short Papers (6min presentations + 2 min questions) \& Industry Session

Time Sub. ID

Contribution Title | Presenter Family Name / First Name / Presenter Affiliation

Country

11:20-11:28 $256 \quad$ Knowledge Sharing in Digital Learning Communities: A Comparative Review of Issues between Education and Industry

Jackson Timothy Michael University of Wollongong Australia

11:30-11:38 $343 \quad$ Adopting Good-Learners' Paths in an Intelligent Tutoring System

Meliana Selly Telkom University Indonesia

11:40-11:48 $\quad 160 \quad$ Smart Phone Endowed Intelligent Teaching for University General Education Curriculum in China
Liu
Zhen
South China University of Technology
China

11:50-11:58 $186 \quad$ Using Trello to Support Agile and Lean Learning with Scrum and Kanban in Teacher Professional Development

Inkila Milla Nhe Mind Lab New Zealand

12:00-12:08 $149 \quad$ TSP: Truthful Grading-Based Strategyproof Peer Selection for MOOCS

Wang Yufeng Nanjing University of Posts and Telecommunications

12:10-12:30 $503 \quad$ Enhancing Digital Learning in Engineering, Solved

Sofios Haritos Smart Sparrow Australia

Kembla : XR \& Immersive Learning Environments - Short Papers (6min presentations + 2min questions) \& Industry Session

Time

Sub. ID

Contribution Title | Presenter Family Name / First Name / Presenter Affiliation

Country

11:20-11:28 $103 \quad$ Using Virtual Reality to Enhance Learning in a Chinese Architectures Course: A Flipped Classroom Approach
Im
Siu Wo Tarloff
City University of Hong Kong
Hong Kong

$11: 30-11: 38 \quad 249$

AHA: ADHD Augmented (Learning Environment)

Hasegawa Tomonori University College Dublin

Ireland

11:40-11:48 $258 \quad$ Extending a Virtual Reality Nasal Cavity Education Tool with Volume Rendering

Marks Stefan Auckland University of Technology New Zealand

11:50-11:58 $362 \quad$ Why Don't You Evacuate Speedily? Augmented Reality-Based Evacuee Visualisation in ICT-based Evacuation Drill

Mitsuhara Hiroyuki Jokushima University Japan

12:00-12:08 $452 \quad$ An Evaluation of Nursing Student Motivation to Learn through Holographic Mixed Reality Simulation

Hauze Sean San Diego State University USA

12:10-12:18 $481 \quad$ Seeking the Treasures of Theoretical Computer Science Education: Towards Educational Virtual Reality for the Visualization of Finite State Machines

Dengel Andreas University of Passau Germany

12:20-12:33 $327 \quad$ The Potential of Augmented Reality for Computer Science Education (Full paper 10min presentation +3 min questions)
Resnyansky
Dmitry
University of South Australia
Australia

Keira : Industry Session

Time Sub. ID

Contribution Title | Presenter Family Name / First Name / Presenter Affiliation

Country

11:20-12:00 $514 \quad$ The Future of Industry and Preparing the Future Workforce

Dodd Brenton Solvia (Dassault Systèmes Business Solutions) Australia

Belmore : Special Session

Time Sub. ID

Contribution Title | Presenter Family Name / First Name / Presenter Affiliation

Country

11:20-12:40 $468 \quad$ Practice-Based Simulation to Develop Students into Engineering Cadets

Devitt Joshua Charles Sturt University

Australia 


\section{Throsby (Ballroom 1) : Engineering Education - Short Papers (6min presentations +2 min questions)}

Time

Sub. ID

Contribution Title | Presenter Family Name / First Name / Presenter Affiliation

Country

13:40-13:48 $307 \quad$ Praxis-Oriented Teaching of Project Management Skills for STEM Students in Higher Education

Rich $\quad$ Beatrice M. $\quad$ Brandenburg University of Technology

Germany

13:50-13:58 $308 \quad$ Preparing STEM Students for Leading Positions through Supervising Younger Students

Worlitz Jane Brandenburg University of Technology

Germany

$14: 00-14: 08 \quad 340$

'Learning 4.0': A Conceptual Discussion

Klopp Marco Aschaffenburg University of Applied Sciences

Germany

Development of a Real-Time Evaluation Support System Using Physiological Index: Case Study of a Simulator-Based Ship Handling Exercise

Murai Koji Tokyo University of Marine Science and Technology Japan

14:20-14:28 $400 \quad$ The Impact of Final Term Exam Exemption Policy: Case Study at MAAP

David Lawrence C. G. Maritime Academy of Asia and the Pacific Philippines

14:30-14:38 $377 \quad$ A Preliminary Study of the Implementation of Concept Maps for Teaching and Learning Strategies in a Power Engineering Course

Aziz Anees Binti Abdul Universiti Teknologi MARA

Malaysia

14:40-14:48 $392 \quad$ Empowering Weak Engineering Students through My UiTM Engineer Program and Understanding the Characteristics of Weak Students
Hussin
Hanim
Universiti Teknologi MARA
Malaysia

$14: 50-14: 58 \quad 179$

Multidisciplinary Student Performance-Based Teaching Design for Engineering Design via a Commercial Case

Liu

Zhen

South China University of Technology

China

\section{McCabe (Ballroom 2) : Miscellaneous Short Papers - Short Papers (6min presentations +2 min questions)}

Time

Sub. ID

\section{Contribution Title | Presenter Family Name / First Name / Presenter Affiliation}

Country

13:40-13:48 $472 \quad$ Engineering Students at Day Zero: Selection and Concerns by Gender

Nikolic Sasha University of Wollongong

Australia

13:50-13:58 $\quad 143 \quad$ Towards a Holistic Approach to Improve the Retention Rate of Freshmen in Engineering

Abid

Muhammad Irfan Riphah International University, Faisalabad

Pakistan

Readiness of Japanese Elementary School Teachers to Begin Computer-Programming Education

Ohashi $\quad$ Yutaro Nippon Institute of Technology

Japan

The Beneficial Effects of an Autonomously Operated Engineering Education Outreach Program: A Case Study of the Servant Leadership Program 
13:40-13:48 $376 \quad$ A Blended Learning Approach to Experiential STEM Education for Young Learners

$\mathrm{Ng} \quad$ David C Shantou University $\quad$ China

13:50-13:58 $\quad 410 \quad$ Quality Assurance of a Hybrid Online Course through Iterative Process Control

Wan Han Beihang University China

14:00-14:08 $\quad 426 \quad$ Teaching Students about Machine Learning through a Gamified Approach

Rattadilok Prapa University of Nottingham Ningbo China China

14:10-14:18 $459 \quad$ Towards Smart Educational Recommendations with Reinforcement Learning in Classroom

Hei Xiaojun Huazhong University of Science and Technology China

14:20-14:28 $330 \quad$ Blended Learning for the Indo-Pacific

Worthington Tom Australian National University Australia

14:30-14:38 $351 \quad$ The Use of Multiple-Choice Questions in 3rd-Year Electronic Engineering Assessment: A Case Study

Bremner Duncan James University of Glasgow UK

14:40-14:48 $433 \quad$ International Experiences and the Implications for the Success of Engineering Programs

Vasconcelos $\quad$ Rosa M. University of Minho

Portugal

\section{Kembla : XR \& Immersive Learning Environments - Short Papers (6min presentations + 2min questions)}

\section{Time \\ Sub. ID \\ Contribution Title | Presenter Family Name / First Name / Presenter Affiliation}

Country

13:40-13:48 $133 \quad$ Designing Immersive Mobile Mixed Reality for Paramedic Education

Cochrane Thomas Donald Auckland University of Technology

New Zealand

13:50-13:58 $\quad 180 \quad$ Embedding Mixed Reality in Humanitarian Logistics Gaming

William Linda National University of Singapore Singapore

14:00-14:08 $368 \quad$ A Novel Augmented Reality Guidance System for Future Informatization Experimental Teaching

Cao Yanpeng Southeast University China

14:10-14:18 $373 \quad$ An Experimental Research of Augmented Reality Technology From the Perspective of Mobile Learning

Cai Wu South China Normal University China

14:20-14:28 $451 \quad$ Design and Practice of Exploratory Virtual Experiment in Physics Discipline

Yu Shufan Central China Normal University China

14:30-14:38 $497 \quad$ Integrating an Intelligent Tutoring System with a Dialogue Manager for Procedural Training in a 2D/3D Virtual Environment

Paladines José Universidad Estatal del Sur de Manabí Ecuador

14:40-14:48 $\quad 480 \quad$ Examining the Impact of Pedagogical Agents on Students Learning Expirience in Virtual Worlds

Nikolic Sasha University of Wollongong

Australia

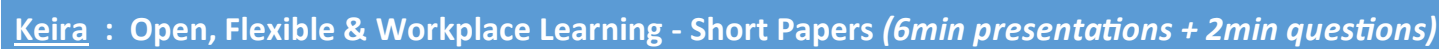

Time Sub. ID

Contribution Title | Presenter Family Name / First Name / Presenter Affiliation

Country

13:40-13:48 $\quad 147 \quad$ Characterizing Concept Conveying in Interactions between MOOC Students and Assistants

Wu Xiao-Xiao Central China Normal University

China

13:50-13:58 $454 \quad$ Harnessing OERs in Hong Kong Technical and Vocational Education and Training (TVET)

Towey Dave University of Nottingham Ningbo China

China

$14: 00-14: 08 \quad 471$

Open-Space Teaching and Learning in Tertiary Education: A Case Study

Ramezanianpour Mohammad Ara Institute of Canterbury

New Zealand

14:10-14:18 $488 \quad$ EntreCompOnto: An Ontology for Semantic Representation of Entrepreneurship Competencies

Tovar Edmundo Universidad Politécnica de Madrid $\quad$ Spain

14:20-14:28 $463 \quad$ Surviving in the Gig Economy: Change of STEM Students' Perceptions on the Generic Skills for the Workplace

Mark Kai Pan The Hong Kong Polytechnic University 
Belmore : Special Session

Time Sub. ID

13:40-15:00 $490 \quad$ Online Laboratories in Engineering Education: Innovation, Disruption, and Future Potential
Saliah-Hassane
Hamadou
TÉLUQ University
Canada

13:40-15:00 201

PILAR: A Federation of VISIR Remote Laboratory Systems for Educational Open Activities

Loro

Felix Garcia

UNED, Spain

Spain

Boardroom : Special Session (Part 2)

Time Sub. ID

Contribution Title | Presenter Family Name / First Name / Presenter Affiliation

Country

13:40-15:00 $226 \quad$ Enhancing Class Interactivity - Teaching and Learning Professional Development Session (Part 2)

Dean

Bonnie Amelia

University of Wollongong

Australia

Registration \& Exhibit Area

15:00-15:30 Afternoon Tea $\quad$ Registration \& Exhibit Area

Grand Ballroom

$\begin{array}{lll}\text { 15:34-16:20 } & \text { Keynote by Emeritus Professor James Trevelyan } & \text { Grand Ballroom } \\ \text { 16:20-17:00 } & \text { Awards and Closing Ceremony } & \text { Grand Ballroom }\end{array}$

The Deck

17:00-18:00

Post-Conference Drinks

The Deck

\section{TALE 2018 Organising Committee}

\section{General Co-Chairs}

Sasha Nikolic, University of Wollongong, Australia

Mark J. W. Lee, Charles Sturt University, Australia

\section{Technical Program Chairs}

Montserrat Ros, University of Wollongong, Australia

Gary K. W. Wong, The University of Hong Kong

Neelakantam Venkatarayalu, Singapore Institute of Technology

Henry C. B. Chan, The Hong Kong Polytechnic University

\section{Publication Chairs}

Jun Shen, University of Wollongong, Australia

Leon C. U. Lei, The University of Hong Kong

Dale Carnegie, University of Wellington, New Zealand

\section{Special Sessions, Panels \& Workshops Chair}

Andrea Goncher, Charles Sturt University, Australia

\section{Special Tracks Coordinator}

Neelakantam Venkatarayalu, Singapore Institute of Technology

\section{Finance and Treasury Chair}

Sudipta Chakraborty, Macquarie University, Australia

\section{Publicity Chairs}

Christian Ritz, University of Wollongong, Australia Kai Pan Mark, The Hong Kong Polytechnic University Minjuan Wang, San Diego State University, USA Maiga Chang, Athabasca University, Canada Olaf Hallan Graven, University College of Southeast Norway Urban Burnik, University of Ljubljana, Slovenia

\section{International Co-Chairs}

Greater China - Stephen J. H. Yang, National Central University, Taiwan; Yong-he Wu, East China Normal University, P. R. China Japan and Korea - Hiroyuki Mitsuhara, Tokushima University, Japan

Indian Subcontinent - K. R. V. "Raja" Subramanian, Myanmar Institute of Information Technology, Myanmar and International Institute of Information Technology, Bangalore, India

Southeast Asia - Ford Lumban Gaol, BINUS University, Indonesia

Oceania - Demetrios G. Sampson, University of Piraeus, Greece and Curtin University, Australia; David Tien, Charles Sturt University, Australia

\section{Special Track Chairs}

Special Track on Engineering of Technologies for Learning - Mark J. W. Lee, Charles Sturt University, Australia; Edilson Arenas, Central Queensland University, Australia; Leon C. U. Lei, The University of Hong Kong

Special Track on X-Reality and Immersive Learning Environments - Jonathon Richter, Salish Kootenai College, USA; Minjuan Wang, San Diego State University, USA; Nian -Shing Chen, National Sun Yet-sen University, Taiwan; Mark J. W. Lee, Charles Sturt University, Australia

Special Track on Big Data, Analytics and Machine Learning in Education - Abelardo Pardo, University of South Australia; Vitomir Kovanović, University of South Australia 


\section{Sponsoring IEEE Entities}
(1) 르를
IEEE Education Society
Advancing Technology
IEEE New South Wales Section for Humanity

\section{Sponsoring Co-Host Institutions}

UNIVERSITY
OF WOLLONGONG
AUSTRALIA

\section{)) $\int\left(\begin{array}{l}\text { Charles Sturt } \\ \text { University }\end{array}\right.$}

\section{Gold Sponsor}

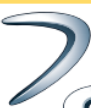

15 DASSAULT | The 3DEXPERIENCE Company
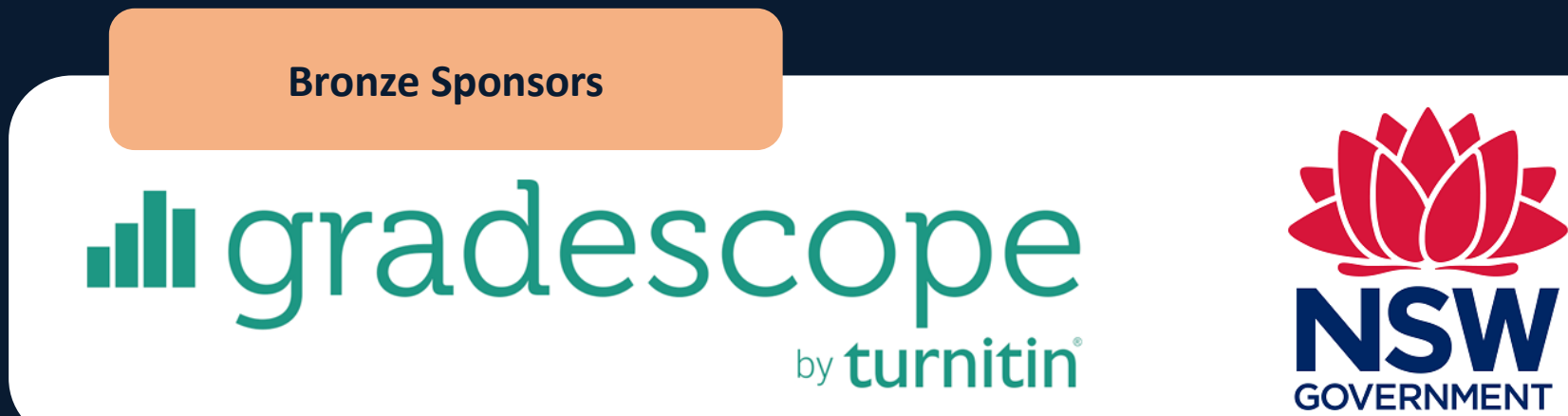

\section{Exhibitors}

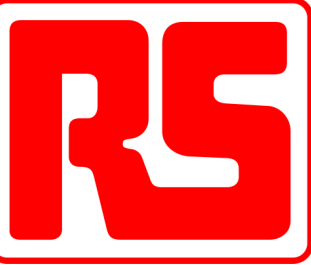

ORACLE

Academy
Korea Institute of

Science and Technology Information
KiSTi www.kisti.re.k
SMART SPARROW 